\title{
COSMOLOGICAL SINGULARITIES IN FLRW SPACETIMES
}

\author{
L. FERNÁNDEZ-JAMBRINA \\ ETSI Navales, Universidad Politecnica de Madrid \\ Arco de la Victoria s/n, E-28040-Madrid, Spain \\ 'E-mail: leonarto fernandezQupm.es \\ www. etsin.upm.es/ufj.htm \\ R. LAZKOZ \\ Frisica Térica, Facultad de Ciencia y Tecrología, \\ Universidad del Fars Vasco, Apdo. 644, E-48080 Bubao, Spain \\ E-maul: ruth.lazkoz@ehu.es \\ tp.dc.ehu.es/RLS.html
}

In this talk we review the appearance of new types of singularities (big rip, sudden singularities...) in FLRW cosmological models that have arisen on considering explanations for accelerated expansion of our universe.

Keyuords: Cosmological singularities, dark energy, geodesics.

\section{Introduction}

During the last decade the observational evidence in favour of accelerated expansion of our universe has increased considerably. ${ }^{1}$ In order to cope with this fact two lines of development have been proposed: either general relativity is to be modified $^{2}$ or our universe is pervaded by an exotic source named dark energy, ${ }^{3}$ which is responsible for the accelerated expansion. Violation of classical energy conditions by this fluid has made feasible a number of new scenarios ${ }^{4}$ for the final fate of our universe (big rip, ${ }^{5}$ sudden singularities, ${ }^{6}$ big brake, ${ }^{7}$ big freeze,${ }^{8}$ inaccesible singularities, ${ }^{9}$ directional singularities, ${ }^{10} w$-singularities, ${ }^{11}$ in braneworld model ${ }^{12} \ldots$ ), which had not been considered previously. In this talk we deal with the appearance and strength of these singular behaviours in FLRW cosmological spacetimes from the point of view of geodesic incompleteness.

\section{Behaviour of singularities}

We choose coordinates for which the FLRW metric is written as

$$
d s^{2}=-d t^{2}+a^{2}(t)\left\{f^{2}(r) d r^{2}+r^{2} d \Omega^{2}\right\}, \quad f^{2}(r)=\frac{1}{1-k r^{2}}, \quad k=0, \pm 1
$$

and we assume that the scale factor $a(t)$ admits a generalised power expansion ${ }^{13}$ in the vicinity of an event at $t_{0}$,

$$
a(t)=c_{0}\left|t-t_{0}\right|^{\eta_{0}}+c_{1}\left|t-t_{1}\right|^{\eta_{1}}+\cdots,
$$

where $\eta_{0}<\eta_{1}<\cdots, c_{0}>0$. Models with ascillatory behaviour ${ }^{14}$ might drop out of this scheme. At lowest order three different qualitative behaviours arise:

- $\eta_{0}>0$ : a vanishing scale factor at to means a Big Bang or Big Crunch. 
- $\eta_{0}=0$ : a finite scale factor at $t_{0}$ means that either $a(t)$ is analytical and the event is regular or on the other hand a weak or sudden singularity appears.

- $\eta_{0}<0$ : a diverging scale factor at $t_{0}$ means a Big Rip singularity.

Geodesics are parametrised by their proper time $\tau,(t(\tau), r(\tau), \theta(\tau), \phi(\tau))$,

$$
t^{\prime}=\sqrt{\delta+\frac{P^{2}}{a^{2}(t)}}, \quad r^{\prime}= \pm \frac{P}{a^{2}(t) f(r)}
$$

may be simplified in terms of a constant of geodesic motion $P$ and $\delta=0,1$ for null and timelike geodesics. The null case $\delta=0$ is straightforwardly solved

$$
a(t) t^{\prime}=P \Rightarrow \int_{t_{0}}^{t} a\left(t^{\prime}\right) d t^{\prime}=P\left(\tau-\tau_{0}\right),
$$

and the geodesic is complete if and only if the scale factor is an integrable function.

Dealing with finite bodies, a singularity is strong ${ }^{15}$ if tidal forces are capable of disrupt them. Following Tipler, a singularity is strong if their volume tends to zero. Folllowing Królak, just a negative derivative of the volume is required. The results are consigned in Table $1 .^{16}$ Conformal diagrams for these models can be found in. ${ }^{17}$

Table 1. Strength of singularities in FLRW models.

\begin{tabular}{cccccc}
\hline$\eta_{0}$ & $\eta_{1}$ & $k$ & $\infty$ & Tipler & Królak \\
\hline$(-\infty, 0)$ & $\left(\eta_{0}, \infty\right)$ & $0, \pm 1$ & $(0, \infty)$ & Strong & Strong \\
0 & $(0,1)$ & $0, \pm 1$ & $(0, \infty)$ & Weak & Strong \\
0 & {$[1, \infty)$} & $0, \pm 1$ & $(0, \infty)$ & Weak & Weak \\
$(0,1)$ & $\left(\eta_{0}, \infty\right)$ & $0, \pm 1$ & $(0, \infty)$ & Strong & Strong \\
1 & $(1, \infty)$ & 0,1 & $(0, \infty)$ & Strong & Strong \\
1 & $(1, \infty)$ & -1 & $(0,1) \cup(1, \infty)$ & Strong & Strong \\
1 & $(1,3)$ & -1 & 1 & Weak & Strong \\
1 & $(3, \infty)$ & -1 & 1 & Weak & Weak \\
$(1, \infty)$ & $\left(\eta_{0}, \infty\right)$ & $0, \pm 1$ & $(0, \infty)$ & Strong & Strong \\
\hline
\end{tabular}

\section{Conclusions}

We have obtained a classification of singular events in FLRW models in terms of their strength and the exponents of an expansion of the scale factor around an event at $t_{0}$. In models with $\eta_{0} \leq-1$, null geodesics avoid the Big Rip singularity. The only models which allow regular behaviour close to $t_{0}$ are those with $\eta_{0}=0$ (de Sitter, sudden...) and with $\eta_{0}=1, k=-1, c_{0}=1$ (Milne).

\section{Acknowledgements}

R.L. is supported by the University of the Basque Country through research grant GIU06/37 and by the Spanish Ministry of Education and Culture through research grant FIS2007-61800. 


\section{References}

1. A. G. Riess et al., Astron. J. 116, 1009 (1998); S. Perlmutter et al., Astrophys. J. 517, 565 (1999); T. M. Davis et al., Astrophys. J. 666, 716 (2007); W. M. Wood-Vasey et al., Astrophys. J. 666, 694 (2007); D. N. Spergel et al. Astrophys. J. Suppl. 148, 175 (2003); D. N. Spergel et al., Astrophys. J. Suppl. 170, 377 (2007).

2. R. Maartens, J. Phys.: Conf. Ser. 68, 012046 (2007);. R. Durrer and R. Maartens, Gen. Rel. Grav. 40, 301 (2008); S. Capozziello and M. Francaviglia, Gen. Rel. Grov. 40, 357 (2008); S. Nojiri and S. D. Odintsov, Int. J. Geom. Meth. Mod. Phys. 4, 115 (2007); A. A. Starobinsky, JETP Lett. 86, 157 (2007); W. Hu and I. Sawicki, Phys. Rev. $D$ 76, 064004 (2007); S. A. Appleby and R. A. Battye, Phys. Lett. B 654, 7 (2007); S. Tsujikawa, Phys. Rev. D 77, 023507 (2008); S. Nojiri and S. D. Odintsov, Phys. Lett. B 652, 343 (2007); S. Nojiri and S. D. Odintsov, Phys. Lett. B 657, 238 (2007); S. Nojiri and S. D. Odintsov, Phys. Rev. D 77, 026007 (2008); S. Capozziello, M. De Laurentis, S. Nojiri, S.D. Odintsov, Phys. Rev. D 79, 124007 (2009); Ii. Bamba, C. Geng, S. Nojiri, S.D. Odintsov, Phys. Rev. D 79,083014 (2009).

3. T. Padmanabhan, AIP Conf. Proc. 861, 179 (2006); A. Albrecht et al., [arXiv:astroph/0609591]; V. Sahni and A. Starobinsky, Int. J. Mod. Phys. D 15, 2105 (2006).

4. S. Nojiri, S. D. Odintsov and S. Tsujikawa, Phys. Rev. D 71, $063004(2005)$.

5. R. R. Calkwell, M. Kamionkowski, N. N. Weinberg, Phys. Ret. Lett. 91, 071301 (2003).

6. J.D. Barrow, Class. Quant. Grav. 21, L79 (2004) ; S. Nojiri, S.D. Odintsov, Phys. Lett. B 595, 1 (2004); J.D. Barrow, Class. Quant. Gruv. 21, 5619 (2004); K. Lake, Class. Quant. Grav. 21, L129 (2004); S. Nojiri, S.D. Odintsov, Phys. Rev. D 70, 103522 (2004); M.P. Dạbrowski, Phys. Rev. D 71, 103505 (2005); J.D. Barrow, C.G. Tsagas, Class. Quont. Gmo, 22, 1563 (2005); L.P. Chimento, R. Lazkoz, Mod. Phys. Lett. A 19, 2479 (2004) ; M.P. Da̧browski, Phys. Lett. B 625, 184 (2005); A. Balcerzak, M.P. Dąbrowski, Phys. Rev. D 73, 101301 (2006); J.D. Barrow, A.B. Batista, J.C. Fabris, S. Houndjo, Phys. Rev. D 78, 123508 (2008); J.D. Barrow, S.Z.W. Lip, Phys. Rev. $D$ 80, 043518 (2009); S. Nojiri, S.D. Odintsov, Phys. Rev. D 78, 046006 (2008); M. R. Setare, E. N. Saridakis, Phys. Lett. B 671, 331 (2009).

7. V. Gorini, A.Y. Kamenshchik, U. Moschella, V. Pasquier, PRD 69, 123512 (2004).

8. M. Bouhmadi-López, P. F. Gonzalez-Díaz and P. Martín-Moruno, Phys. Lett. B 659, 1 (2008).

9. B. McInnes, Class. Quant. Gruv. 24, $1605(2007)$.

10. L. Fernández-Jambrina, Phys. Lett. B 6569 (2007) [arXiv:gr-qc/0704.3936]

11. M.P. Dabrowski, T. Denkiewicz, Phys. Rev. D 79, 063521 (2009); L. FernándezJambrina, (in preporation).

12. Y. Shtanov, V. Sahni, Class. Quant. Grav. 19, L101 (2002); R. A. Brown, R. Maartens, E. Papantonopoulos and V. Zamarias, JCAP 0511, 008 (2005); P. Tretyakov, A. Toporensky, Y. Shtanov and V. Sahni, Class. Quant. Grav. 23, 3259 (2006).

13. C. Cattoën, M. Visser, Class. Quant Grov. 22, 4913 (2005); C. Cattoën, MSc thesis, Victoria University of Wellington (2006).

14. T. Cailleteau, A. Cardoso, K. Vandersloot, D. Wands, [arXiv: 0808.0190v2].

15. G.F.R. Ellis and B.G. Schmidt, Gen. Rel. Grav, 8, 915 (1977); F.J. Tipler, Phys. Lett. A 64, 8 (1977); A. Królak, Class. Quant. Grvev. 3, 267 (1986); C.J.S. Clarke and A. Królak, Journ. Geom. Phys. 2, 127 (1985).

16. L. Fernández-Jambrina and R. Lazkoz, Phys. Rev. D 70, 121503 (2004) [arXiv:grqc/0410124]; L. Fernández-Jambrina, R. Lazkoz, Phys. Lett. B 670, 254-258 (2009) [arXiv:0805.2284]; L. Fernández-Jambrina and R. Lazkoz, Phys. Rev. D 74, 064030 (2006) [arXiv:gr-qc/0607073]; L. Fernández-Jambrina and R. Lazkoz, (in preparation).

17. P.A. Hohn, S.M. Scott, Class. Quant. Grav. 26, 035019 (2009). 